\title{
Weed Dynamics in No-Till Rainfed Crops in Chaouia, Semi-Arid Morocco
}

\author{
Abbès Tanji ${ }^{1}$, Oussama El Gharras ${ }^{2}$, Hassan Ouabbou ${ }^{2} \&$ Todorovic Mladen $^{3}$ \\ ${ }^{1}$ Retired Weed Scientist, Settat, Morocco \\ ${ }^{2}$ Institut National de la Recherche Agronomique, Settat, Morocco \\ ${ }^{3}$ Mediterranean Agronomic Institute of Bari, Italy \\ Correspondence: Abbès Tanji, Farah 1 Rue Kacem Amine No. 1, 26002, Settat, Morocco. Tel: \\ 00212-661-234-019. E-mail: abbestanji1@gmail.com
}

Received: July 23, 2017

doi:10.5539/jas.v9n11p198
Accepted: September 13, $2017 \quad$ Online Published: October 15, 2017

URL: https://doi.org/10.5539/jas.v9n11p198

The research is financed by ACLIMAS (Adaptation to Climate Change of the Mediterranean Agricultural Systems) project within the Sustainable Water Integrated Management (SWIM) programme of the European Commission.

\begin{abstract}
Three on-farm research-managed trials were conducted in Settat province, Chaouia, semi-arid Morocco, from 2012-13 to 2014-15, to investigate the dynamics of germinable soil seedbank, weed density, and community composition of weeds in 3 crop rotations: continuous durum wheat, barley + pea/durum wheat/durum wheat, and canola/durum wheat/durum wheat. Initial germinable weed seedbank density estimated in September 2012, before no-till planting in November 2012 was 1890 seeds $\mathrm{m}^{-2}$. After two growing seasons, seedbank reductions were $23 \%$ in continuous durum wheat, $68 \%$ in canola/durum wheat/durum wheat, and $72 \%$ in barley + pea/durum wheat/durum wheat. In continuous durum wheat, weed densities before no-till planting were 273,46 , and 59 plants $\mathrm{m}^{-2}$ in November 2012, December 2013, and November 2014, respectively. In herbicide-free barley + pea/durum wheat/durum wheat, weed densities before no-till planting durum wheat were 128 and 42 plants $\mathrm{m}^{-2}$ in December 2013 and November 2014, respectively. In canola/durum wheat/durum wheat, weed densities before no-till planting wheat were only 20 and 25 plants ${ }^{-2}$ in December 2013 and November 2014, respectively. This study demonstrated the combined merits of pre-plant glyphosate, herbicide use in wheat, herbicide-free barley + pea haying, and durum wheat rotation with either canola or barley + pea to manage weeds in no-till systems in semi-arid Morocco.
\end{abstract}

Keywords: weed seedbank, weed density, crop rotation, no-till, Morocco

\section{Introduction}

Conservation agriculture (CA) is based on minimum soil disturbance, permanent soil cover, and crop rotation; it is promoted as a sustainable alternative to systems involving conventional tillage (Kassam, 2014; Loss et al., 2015; Nichols et al., 2015). In Morocco, much of the CA work done since the early 1980s has shown that yields can be improved with no-till systems (Abail et al., 2013; Belmekki et al., 2014; Boughlala et al., 2011; El Gharras et al, 2010; Labbaci et al., 2015; Moussadek, 2011; Mrabet, 2008; Mrabet et al., 2012; Ramdani et al., 2010; Schwilch et al., 2015). However, CA adoption was quite low in Morocco due to a general perception that no-till farming systems require more herbicides than conventional agriculture (El Brahli \& Mrabet, 2000). Such increased use of herbicides may be considered by small farmers a major problem of no-till farming particularly in a time when the use of agrochemicals is considered by the general public as threat for human health and the environment (Friedrich, 2005).

A number of CA practices designed to replace or improve continuous wheat production intend to introduce nitrogen fixing crops, reduce soil disturbance, and retain surface crop residues (Kassam, 2014). Ultimately, these practices should improve crop yields, reduce costs of crop production, increase soil organic matter content, and improve long-term soil health. However, adoption of CA is often hindered by farmers' limited understanding of the changes in weed control practices and crop performance during the transition period. Integrated weed 
management under CA may include the combination of applied soil herbicides, crop rotation, and competitive crop varieties with weeds (Anderson, 2015; Blackshaw et al., 2005, 2015; Gulden et al., 2011; Harker \& O’Donovan, 2013; Holm et al., 2006).

In crop/livestock integrated production systems, weed seeds are an important challenge since most weeds are considered as free forage for livestock and the weedy fallow is widely practiced (El Housni et al., 2013). No-till systems accumulate seeds near the soil surface where they are more likely to germinate but are also exposed to greater mortality risks through weather variability and predation. Assuming no seed input into the system, germinable seedbanks under no-till decrease more rapidly than under conventional tillage. Reducing tillage may shift seedbank densities and weed communities from annual broadleaves to annual grasses and/or perennials (Aibar, 2006; El Brahli \& Mrabet, 2000; Gulden et al., 2011; Jose Maria \& Sans, 2011; Murphy et al., 2006; Nichols et al., 2015; Recasens et al., 2016).

Under semi-arid Morocco conditions, our knowledge of the effects of crop rotation and herbicides on weed seedbank community size and structure is limited. Weed seedbank densities have been studied less frequently than weed plant communities because they require labor and greenhouse. Tanji et al. (2017) reported that integrated weed management combining glyphosate before no-till planting, post-emergence herbicide use in bread wheat, haying barley + pea mixture, within the crop rotation (barley + pea/bread wheat) reduced weed seedbank by up to $35 \%$, species richness by up to $47 \%$, and weed density prior to wheat harvest or forage haying by up to $83 \%$. In this paper, we provide results from recent 3 on-farm trials related to the combined effects of crop rotation, pre-plant glyphosate, herbicide use in wheat, and herbicide-free barley + pea mixture on the weed seedbank and weed density.

\section{Materials and Methods}

\subsection{General}

On-farm trials were conducted from 2012-13 to 2014-15 in the Settat province, Chaouia, Morocco. The climate is semi-arid, and precipitations during the 3 cropping seasons varied from $232 \mathrm{~mm}$ to $455 \mathrm{~mm}$ with more than $60 \%$ occurring from November through April (Table 1). Under Mediterranean conditions, monthly minimal temperatures are usually 5 to $10{ }^{\circ} \mathrm{C}$ from December to February and monthly maximal temperatures are 25 to $30{ }^{\circ} \mathrm{C}$ from June to August. The soil is a vertisol with $55 \%$ clay.

Three farms were therefore selected in 2012 and 3 hectares (ha) in each farm were used to conduct no-till research-managed trials. One ha was no-till planted to durum wheat (Triticum durum Desf.), 1 ha was no-till planted to a forage mixture of barley (Hordeum vulgare L.) + pea (Pisum sativum L.), and 1 ha to canola (Brassica napus L.). Three crop rotations were managed using conservation management practices: continuous durum wheat, barley + pea/durum wheat/durum wheat, and canola/durum wheat/durum wheat. Prior to 2012-13, all fields have been planted with wheat or barley using conventional system.

Table 1. Monthly precipitation at Settat, Morocco, from 2012-13 to 2014-15

\begin{tabular}{llll}
\hline Month & $2012-13$ & $2013-14$ & $2014-15$ \\
\hline September & 23.4 & 6.8 & 9.5 \\
October & 124.0 & 1.6 & 6.6 \\
November & 91.2 & 27.7 & 164.0 \\
December & 6.5 & 18.4 & 50.3 \\
January & 36.0 & 98.4 & 55.9 \\
February & 20.5 & 27.8 & 8.9 \\
March & 111.5 & 9.5 & 57.9 \\
April & 41.6 & 42.1 & 0 \\
May & 0 & 0 & 15.5 \\
June & 0 & 0 & 0.0 \\
July & 0 & 0 & 0 \\
August & 0 & 0 & 377.6 \\
\hline Total & 454.7 & 232.3 & \\
\hline
\end{tabular}


"Karim" durum wheat variety and local barley and pea seeds were no-till planted in November 2012, 2013, and 2014. The wheat crop was fertilized at $100 \mathrm{~kg} \mathrm{ha}^{-1}$ of diammonium phosphate (18-46-0) and no-till planted at $120 \mathrm{~kg} \mathrm{ha}^{-1}$ of seeds. The forage mixture was fertilized the same way as the durum wheat and no-till planted at $25+75 \mathrm{~kg} \mathrm{ha}^{-1}$ of barley + pea seeds. Canola was fertilized the same way as the wheat and forage mixture and no-till planted at $3 \mathrm{~kg}$ of seeds ha ${ }^{-1}$. Top dressing nitrogen was used at wheat tillering at the rate of $33 \mathrm{~kg} \mathrm{ha}^{-1}$. The utilized no-till seeder is a tine seed drill of $18 \mathrm{~cm}$ row spacing.

At wheat tillering, the broadleaf herbicide florasulam $+2,4-\mathrm{D}\left(3.75+180 \mathrm{~g} \mathrm{ha}^{-1}\right)$ was used in durum wheat fields. A foliar fungicide (epoxyconazol at $125 \mathrm{~g} \mathrm{ha}^{-1}$ ) was sprayed on wheat at the booting stage. Herbicides and fungicides were sprayed with a tractor-mounted sprayer, and volumes of spray were $120 \mathrm{~L} \mathrm{ha}^{-1}$ for herbicides and $200 \mathrm{~L} \mathrm{ha}^{-1}$ for fungicide. Glyphosate $\left(720 \mathrm{~g} \mathrm{ha}^{-1}\right)$ was applied in all fields prior to no-till planting.

\subsection{Seedbank Assessment}

The procedure used in the Oued Zem study (Tanji et al., 2017) was used in this study. Each year, ten soil cores (7.2-cm diameter by $10 \mathrm{~cm}$ deep) were randomly collected from each field, bulked, air dried, placed in polyethylene bags, and stored in the greenhouse at room temperature $\left(25 / 10{ }^{\circ} \mathrm{C}\right)$ until November. Germinable weed seedbank determinations were conducted using the greenhouse emergence method (Blackshaw et al., 2005; Gulden et al., 2011; Smith \& Gross, 2006; Tanji et al., 2017). Soil was spread onto plastic trays, placed in a greenhouse with a day/night temperature of $25 / 10{ }^{\circ} \mathrm{C}$ and watered as necessary to keep moist. Weed emergence counts were made twice a month from November to April. Seedlings that emerged were identified and removed. Total seedling counts in each soil sample, from November to April, were considered the germinable fraction of the weed seedbank.

\subsection{Weed Density}

Weed densities were estimated in 10 randomly chosen $\left(0.5 \mathrm{~m} \times 0.5 \mathrm{~m}=0.25 \mathrm{~m}^{2}\right)$ quadrats in each farmer's field at 3 periods (Tables 3 to 5):

> in November or December prior to application of glyphosate $\left(720 \mathrm{~g} \mathrm{ha}^{-1}\right)$ at crop planting,

$>\quad$ in January or February prior to Florasulam $+2,4-\mathrm{D}\left(3.75+180 \mathrm{~g} \mathrm{ha}^{-1}\right)$ application at wheat tillering, and

$>\quad$ in March or April prior to forage haying and wheat and canola harvests.

\subsection{Satistical Analysis}

This study was conducted in 3 farmers' fields in the same region, and each field was considered as one site or one replicate. There were 3 different crop rotations at each site (canola/durum wheat/durum wheat, barley + pea/durum wheat/durum wheat, and continuous durum wheat). Data presented for weed seedbank were means of the 3 sites. Mean weed densities were presented for each crop. Means +/- standard error were calculated using PROC UNIVARIATE with SAS software (SAS Institute v. 9.2, Cary, NC).

\section{Results and Discussions}

\subsection{Weed Seedbank Diversity}

The greenhouse germination study showed that the initial germinable soil weed seedbank from 3 farmers' fields was 1890 seeds $\mathrm{m}^{-2}$ (Table 2) in September 2012. Such high seed density might be due to the lack of appropriate weed management in the traditional cereal/cereal rotation. In fact, farmers were not familiar with herbicide use, and weeds in cereal fields were hand pulled for animal feed.

In September 2013, weed seedbank was 1009 seeds $\mathrm{m}^{-2}$ after one season of durum wheat (in continuous durum wheat), 719 seeds $\mathrm{m}^{-2}$ after barley + pea (in durum wheat/barley + pea rotation), and 564 seeds $\mathrm{m}^{-2}$ after canola (in durum wheat/canola rotation). In comparison to the initial seedbank that was estimated in September 2012 to 1890 seeds $\mathrm{m}^{-2}$, seedbank reductions in the first year of this no-till study were 47,62 , and $70 \%$ after durum wheat, barley + pea forage, and canola, respectively.

In September 2014, seedbank in durum wheat was 1445 seeds $\mathrm{m}^{-2}$ after 2 growing seasons of durum wheat, while durum wheat after canola had 606 seeds $\mathrm{m}^{-2}$, and durum wheat after barley + pea had only $520 \mathrm{seeds}^{-2}$. Compared to the initial seedbank (1890 seeds $\mathrm{m}^{-2}$ ) estimated in September 2012, seedbank reductions after two cropping seasons were $23 \%$ in continuous durum wheat, $68 \%$ in the durum wheat after canola, and $72 \%$ in durum wheat after barley + pea mixture. Nichols et al. (2015) reported that burial increases weed seed survival, while seeds on or close to the soil surface can lose viability due to desiccation and harsh weather. Rotation designs involving four years or more have been shown to drastically reduce weed density and herbicide use in 
both tilled and un-tilled systems (Anderson, 2015; Blackshaw et al., 2005, 2015; Murphy et al., 2006; Stone et al., 2006; Tuesca et al., 2001).

Table 2. Germinable weed seed density in no-till crops in 3 sites in Chaouia, Morocco, in September 2012, 2013, and 2014

\begin{tabular}{|c|c|c|c|c|c|c|c|}
\hline \multirow{4}{*}{ Weed species } & \multirow{2}{*}{$\begin{array}{l}\text { Weed seedbank in } \\
\text { September } 2012\end{array}$} & \multicolumn{3}{|c|}{$\begin{array}{c}\text { Weed seedbank } \\
\text { in September } 2013\end{array}$} & \multicolumn{3}{|c|}{$\begin{array}{c}\text { Weed seedbank } \\
\text { in September } 2014\end{array}$} \\
\hline & & Rotation 1 & Rotation 2 & Rotation 3 & Rotation 1 & Rotation 2 & Rotation 3 \\
\hline & $\begin{array}{l}\text { Conventional } \\
\text { durum wheat } \\
\text { in 2011-12 }\end{array}$ & $\begin{array}{l}\text { durum } \\
\text { wheat in } \\
2011-12 \\
\text { /durum } \\
\text { wheat in } \\
\text { 2012-13 }\end{array}$ & $\begin{array}{l}\text { durum } \\
\text { wheat in } \\
2011-12 \\
\text { /barley + } \\
\text { pea in } \\
2012-13\end{array}$ & $\begin{array}{l}\text { durum } \\
\text { wheat in } \\
2011-12 \\
\text { /canola in } \\
2012-13\end{array}$ & $\begin{array}{l}\text { durum } \\
\text { wheat in } \\
2011-12 \\
\text { /durum } \\
\text { wheat in } \\
2012-13 \\
\text { /durum } \\
\text { wheat in } \\
\text { 2013-14 }\end{array}$ & $\begin{array}{l}\text { durum } \\
\text { wheat in } \\
2011-12 \\
\text { /barley + } \\
\text { pea in } \\
2012-13 \\
\text { /durum } \\
\text { wheat in } \\
\text { 2013-14 }\end{array}$ & $\begin{array}{l}\text { durum } \\
\text { wheat in } \\
2011-12 \\
\text { /canola in } \\
2012-13 \\
\text { /durum } \\
\text { wheat in } \\
\text { 2013-14 }\end{array}$ \\
\hline & \multicolumn{7}{|c|}{ Germinable weed seeds $\mathrm{m}^{-2}$ (average of 3 sites) } \\
\hline Anisantha rigida (Roth) Hyl. & 221 & 224 & 23 & 0 & 676 & 121 & 0 \\
\hline Papaver rhoeas $\mathrm{L}$. & 205 & 203 & 82 & 152 & 348 & 236 & 206 \\
\hline Lysimachia arvensis (L.) U.M.\&A. & 306 & 39 & 36 & 24 & 18 & 18 & 73 \\
\hline Misopates orontium (L.) Raf. & 65 & 55 & 14 & 6 & 112 & 24 & 97 \\
\hline Melilotus sulcatus Desf. & 49 & 3 & 5 & 152 & 3 & 0 & 48 \\
\hline Lolium rigidum Gaud. & 95 & 3 & 0 & 0 & 36 & 0 & 6 \\
\hline Calendula stellata Cav. & 27 & 73 & 45 & 6 & 52 & 18 & 0 \\
\hline Cichorium intybus $\mathrm{L}$. & 11 & 12 & 0 & 0 & 0 & 12 & 0 \\
\hline Scorpiurus muricatus L. & 5 & 33 & 5 & 6 & 18 & 12 & 0 \\
\hline Vaccaria hispanica (Mill.) R. & 8 & 3 & 9 & 30 & 6 & 0 & 12 \\
\hline Medicago polymorpha $\mathrm{L}$. & 44 & 12 & 14 & 0 & & & \\
\hline Plantago afra $\mathrm{L}$. & 3 & 55 & 18 & 0 & & & \\
\hline Diplotaxis tenuisiliqua Delile & 38 & 0 & 68 & 0 & & & \\
\hline Emex spinosa (L.) Campd. & 14 & & & & 3 & 0 & 12 \\
\hline Chenopodium murale $\mathrm{L}$. & 76 & & & & & & \\
\hline Avena sterilis $\mathrm{L}$. & 33 & & & & & & \\
\hline Glebionis coronaria $(\mathrm{L}$.$) Spach$ & 30 & & & & & & \\
\hline Anacyclus maroccanus Ball & 22 & & & & & & \\
\hline Malva parviflora $\mathrm{L}$. & 19 & & & & & & \\
\hline Senecio vulgaris $\mathrm{L}$. & 16 & & & & & & \\
\hline Diplotaxis assurgens (Del.) T. & 14 & & & & & & \\
\hline Rhagadiolus stellatus (L.) G. & 11 & & & & & & \\
\hline Spergula fallax (Lowe) E.H.L.K. & 11 & & & & & & \\
\hline Eryngium ilicifolium Lam. & 8 & & & & & & \\
\hline Glaucium corniculatum (L.) J.H.R. & 8 & & & & & & \\
\hline Heliotropium europaeum $\mathrm{L}$. & 8 & & & & & & \\
\hline Ridolfia segetum (Guss.) Moris & 5 & & & & & & \\
\hline Convolvulus arvensis $\mathrm{L}$. & 3 & & & & & & \\
\hline Ecballium elaterium (L.) A.R. & 3 & & & & & & \\
\hline Others & 532 & 294 & 400 & 188 & 167 & 79 & 152 \\
\hline Total $\left(\right.$ seeds $\left.\mathrm{m}^{-2}\right)$ & 1890 & 1009 & 719 & 564 & 1445 & 520 & 606 \\
\hline Standard error & 488 & 269 & 333 & 136 & 519 & 143 & 170 \\
\hline
\end{tabular}


The canola/durum wheat and barley + pea/durum wheat crop rotations contributed to the rapid decrease in the readily germinable fraction of weed seedbank compared with continuous durum wheat. Higher seedbank reductions in these two crop rotations were essentially due to glyphosate pre-plant and florasulam $+2,4-\mathrm{D}$ post-emergence in wheat. In fact, herbicides were found to be among the main factors controlling arable seedbanks, because they limit both weed growth and weed seed production (Jose Maria \& Sans, 2011; Tanji et al., 2017).

The crop rotation as a weed management strategy showed that starting with canola or a forage mixture reduced the seedbank gradually while starting with a cereal crop showed an increase in rigid brome (Anisantha rigida) seed in the soil seedbank. Rigid brome seedbank density was 221 seeds $\mathrm{m}^{-2}$ in September 2012 and 676 seeds $\mathrm{m}^{-2}$ in September 2014. Therefore, in barley + pea/durum wheat and canola/durum wheat rotations, rigid brome seedbank diminished while continuous durum wheat increased three-fold rigid brome seedbank density in two seasons. In no-tilled wheat fields in Meknès, North Central of Morocco, Hamal et al. (2001) found 876 seeds of rigid brome $\mathrm{m}^{-2}$. Recasens et al. (2016) found that Bromus diandrus density increased in no-till crop rotation of barley/bread wheat/barley during three seasons. However, greater rotational crop diversity resulted in easier weed management (Nichols et al., 2015).

In September 2012, more than 29 weed species were identified in soil seedbank (Table 2). The 5 major weeds were Anisantha rigida, Lysimachia arvensis, Melilotus sulcatus, Misopates orontium, and Papaver rhoeas. More than 16 weed species were not found in September 2013 and September 2014. For example, seedbank decreased for Chenopodium murale, Cichorium intybus, Diplotaxis tenuisiliqua, Emex spinosa, Glebionis coronaria, Lysimachia arvensis, and Melilotus sulcatus. However, seed of Anisantha rigida, Calendula stellata, Misopates orontium, Papaver rhoeas, and Scorpiurus muricatus increased. In a crop rotation study, Lutman et al. (2002) reported that annual seed loss rates varied from 20 to $99 \%$ for 15 weed species.

\subsection{Weed Diversity in Wheat Fields}

In continuous durum wheat, weed densities before applying glyphosate and planting were 273,46 , and 59 plants $\mathrm{m}^{-2}$ in November 2012, December 2013, and November 2014, respectively (Table 3). Compared to the initial weed density ( 273 plants $\mathrm{m}^{-2}$ recorded in November 2012), reductions in weed densities were 83 and $78 \%$ after one and two seasons, respectively. Glyphosate sprayed before or at planting at $720 \mathrm{~g} \mathrm{ha}^{-1}$ provided excellent control of volunteer crops and annual weeds. Perennial weeds such as Arisarum vulgare, Launaea nudicaulis, and Silene vulgaris were partially controlled with glyphosate. Weed densities before herbicide treatments in durum wheat were 115, 25, and 36 plants $\mathrm{m}^{-2}$ in January 2013, February 2014, and January 2015, respectively. Weed densities after herbicide treatments were 57, 18, and 49 plants $\mathrm{m}^{-2}$ in April 2013, March 2014, and April 2015 , respectively.

In herbicide-free barley + pea/durum wheat/durum wheat, weed densities before applying glyphosate and planting durum wheat were 128, and 42 plants $\mathrm{m}^{-2}$ in December 2013 and November 2014, respectively (Table 4). Glyphosate sprayed before or at planting provided excellent control of volunteer crops and annual weeds. After durum wheat emergence, weed densities were 101 and 15 plants $\mathrm{m}^{-2}$ in February 2014 and January 2015, respectively. Weed densities after herbicide treatments in durum wheat were 59 and 10 plants $\mathrm{m}^{-2}$ in March 2014 and April 2015, respectively. In barley + pea forage mixture/durum wheat rotation, volunteer barley and pea were among weeds found in wheat plots.

In canola/durum wheat/durum wheat, weed densities before applying glyphosate and planting wheat were 20 and 25 plants $\mathrm{m}^{-2}$ in December 2013 and November 2014, respectively (Table 5). Pre-plant glyphosate provided excellent weed control. After wheat emergence, weed densities were 27 and 22 plants $\mathrm{m}^{-2}$ in February 2014 and January 2015, respectively. Weed densities after herbicide application in wheat were 12 and 6 plants $\mathrm{m}^{-2}$ in March 2014 and April 2015, respectively.

After crop emergence, 97 weed species were identified in all durum wheat plots during the 3 cropping seasons (Table 3). Major weed species found in durum wheat were Anisantha rigida, Calendula stellata, Diplotaxis tenuisiliqua, Malva parviflora, Melilotus sulcatus, Scorpiurus muricatus, volunteer barley, and volunteer pea. Weed control with postemergence herbicides florasulam + 2,4-D provided excellent control of annual broadleaf weeds in wheat.

Weed species that had low densities in durum wheat in the second and/or third year of various crop rotations included Bupleurum lancifolium, Calendula stellata, Cichorium intybus, Lysimachia arvensis, Melilotus sulcatus, Misopates orontium, Papaver rhoeas, etc. However, rigid brome (Anisantha rigida) had increased density in the second and third year of no-till, essentially in the continuous durum wheat. No-till systems favor species that can successfully germinate on the soil surface such as annual grasses (Nichols et al., 2015; Recasens et al., 2016). 
Table 3. Weed density in continuous no-till durum wheat in 3 sites in Chaouia, Morocco, from 2012-13 to 2014-15

\begin{tabular}{|c|c|c|c|c|c|c|c|c|c|}
\hline \multirow{6}{*}{ Weed species } & \multicolumn{9}{|c|}{ Rotation 1: continuous durum wheat } \\
\hline & \multicolumn{3}{|c|}{$2012-13$} & \multicolumn{3}{|c|}{$2013-14$} & \multicolumn{3}{|c|}{$2014-15$} \\
\hline & \multicolumn{9}{|c|}{ Date of weed density measurement } \\
\hline & $16 \mathrm{Nov}$ & 17 Jan & $16 \mathrm{Apr}$ & $24 \mathrm{Dec}$ & $9 \mathrm{Feb}$ & $8 \mathrm{Mar}$ & $24 \mathrm{Nov}$ & $15 \mathrm{Jan}$ & $1 \mathrm{Apr}$ \\
\hline & 2012 & 2013 & 2013 & 2013 & 2014 & 2014 & 2014 & 2015 & 2015 \\
\hline & \multicolumn{9}{|c|}{ Plants $\mathrm{m}^{-2}$ (average of 3 sites) } \\
\hline Anisantha rigida (Roth) Hyl. & 58 & 7 & 5 & 9 & 5 & 15 & 11 & 10 & 45 \\
\hline Melilotus sulcatus Desf. & 3 & 10 & 2 & 4 & 6 & 0 & 0 & 0 & 0 \\
\hline Hordeum vulgare $\mathrm{L}$. (barley) & 0 & 0 & 0 & 0 & 2 & 1 & 17 & 2 & 1 \\
\hline Calendula stellata Cav. & 1 & 4 & 1 & 12 & 0 & 0 & 0 & 0 & 0 \\
\hline Scorpiurus muricatus L. & 3 & 9 & 13 & 0 & 3 & 0 & 0 & 6 & 2 \\
\hline Avena sterilis $\mathrm{L}$. & 48 & 9 & 17 & 0 & 0 & 0 & 0 & 0 & 0 \\
\hline Papaver rhoeas $\mathrm{L}$. & 17 & 23 & 2 & 0 & 0 & 0 & 0 & 1 & 0 \\
\hline Arisarum vulgare Targ.-Tozz. & 8 & 4 & 1 & 1 & 1 & 0 & 0 & 2 & 0 \\
\hline Cichorium intybus $\mathrm{L}$. & 29 & 4 & 0 & 2 & 0 & 0 & 1 & 0 & 0 \\
\hline Lolium rigidum Gaud. & 17 & 3 & 1 & 0 & 0 & 0 & 0 & 0 & 0 \\
\hline Pisum sativum L. (pea) & 0 & 0 & 0 & 3 & 0 & 0 & 0 & 0 & 0 \\
\hline Lysimachia arvensis (L.) U. M. \& A. & 25 & 2 & 3 & 0 & 0 & 0 & 0 & 0 & 0 \\
\hline Rhagadiolus stellatus (L.) Gaertn. & 2 & 4 & 0 & 1 & 0 & 0 & 0 & 0 & 0 \\
\hline Glebionis coronaria (L.) Spach & 1 & 0 & 1 & 0 & 0 & 0 & 0 & 0 & 0 \\
\hline Geropogon hybridus L. & 2 & 2 & 1 & 0 & 0 & 0 & 0 & 0 & 0 \\
\hline Silene vulgaris (Moench) Garcke & 1 & 1 & 0 & 2 & 0 & 1 & 1 & 0 & 0 \\
\hline Vaccaria hispanica (Mill.) Rauschert & 1 & 4 & 0 & 0 & 1 & 0 & 0 & 0 & 0 \\
\hline Malva parviflora $\mathrm{L}$. & 1 & 0 & 0 & 0 & 0 & 0 & 13 & 0 & 0 \\
\hline Diplotaxis tenuisiliqua Delile & 1 & 0 & 0 & 1 & 0 & 0 & 4 & 6 & 0 \\
\hline Calendula arvensis $\mathrm{L}$. & 2 & 1 & 0 & 0 & 0 & 0 & 1 & 0 & 0 \\
\hline Cynodon dactylon (L.) Pers. & 4 & 0 & 1 & 0 & 0 & 0 & 0 & 0 & 0 \\
\hline Sonchus oleraceus L. & 2 & 1 & 0 & 3 & 2 & 0 & 0 & 0 & 0 \\
\hline Convolvulus althaeoides $\mathrm{L}$. & 1 & 1 & 0 & 3 & 0 & 0 & 3 & 0 & 0 \\
\hline Sinapis arvensis $\mathrm{L}$. & 7 & 0 & 0 & 1 & 0 & 0 & 0 & 0 & 0 \\
\hline Ecballium elaterium (L.) A. Richard & 0 & 0 & 0 & 0 & 1 & 0 & 0 & 0 & 1 \\
\hline Bupleurum lancifolium Hornem. & 0 & 7 & 3 & 0 & 1 & 0 & 0 & 0 & 0 \\
\hline Vicia sativa $\mathrm{L}$. & 0 & 1 & 0 & 0 & 1 & 1 & 0 & 0 & 0 \\
\hline Medicago polymorpha $\mathrm{L}$. & 0 & 0 & 0 & 0 & 0 & 0 & 1 & 6 & 0 \\
\hline Triticum aestivum L. (bread wheat) & 1 & 0 & 0 & 0 & 0 & 0 & 0 & 0 & 0 \\
\hline Misopates orontium (L.) Raf. & 2 & 1 & 0 & 0 & 0 & 0 & 0 & 0 & 0 \\
\hline Convolvulus arvensis $\mathrm{L}$. & 1 & 1 & 1 & 0 & 0 & 0 & 0 & 0 & 0 \\
\hline Plantago afra $\mathrm{L}$. & 1 & 1 & 0 & 0 & 0 & 0 & 0 & 0 & 0 \\
\hline Silybum marianum (L.) Gaertn. & 2 & 1 & 1 & 0 & 0 & 0 & 0 & 0 & 0 \\
\hline Euphorbia exigua L. & 4 & 1 & 0 & 0 & 0 & 0 & 0 & 0 & 0 \\
\hline Galium verrucosum Huds. & 0 & 3 & 0 & 0 & 0 & 0 & 0 & 0 & 0 \\
\hline Emex spinosa (L.) Campd. & 2 & 1 & 0 & 0 & 0 & 0 & 0 & 0 & 0 \\
\hline Phalaris minor Retz. & 2 & 2 & 0 & 0 & 0 & 0 & 0 & 0 & 0 \\
\hline Teucrium resupinatum Desf. & 3 & 0 & 0 & 0 & 0 & 0 & 0 & 0 & 0 \\
\hline Other weeds & 21 & 7 & 4 & 4 & 2 & 0 & 7 & 3 & 0 \\
\hline Total (plants $\mathrm{m}^{-2}$ ) & 273 & 115 & 57 & 46 & 25 & 18 & 59 & 36 & 49 \\
\hline Standard error & 97 & 24 & 16 & 12 & 6 & 7 & 22 & 13 & 46 \\
\hline
\end{tabular}


Table 4. Weed density in no-till crops in rotation 2 (barley + pea/durum wheat/durum wheat) in 3 sites in Chaouia from 2012-13 to 2014-15

\begin{tabular}{|c|c|c|c|c|c|c|c|c|c|}
\hline \multirow{6}{*}{ Weed species } & \multicolumn{9}{|c|}{ Rotation 2: barley + pea/durum wheat/durum wheat } \\
\hline & \multicolumn{3}{|c|}{$\begin{array}{l}\text { barley }+ \text { pea } \\
\text { in 2012-13 }\end{array}$} & \multicolumn{3}{|c|}{$\begin{array}{c}\text { durum wheat } \\
\text { in 2013-14 }\end{array}$} & \multicolumn{3}{|c|}{$\begin{array}{l}\text { durum wheat } \\
\text { in 2014-15 }\end{array}$} \\
\hline & \multicolumn{9}{|c|}{ Date of weed density measurement } \\
\hline & $16 \mathrm{Nov}$ & 17 Jan & $2 \mathrm{Apr}$ & $24 \mathrm{Dec}$ & $9 \mathrm{Feb}$ & $8 \mathrm{Mar}$ & $24 \mathrm{Nov}$ & $15 \mathrm{Jan}$ & $1 \mathrm{Apr}$ \\
\hline & 2012 & 2013 & 2013 & 2013 & 2014 & 2014 & 2014 & 2015 & 2015 \\
\hline & \multicolumn{9}{|c|}{ Plants $\mathrm{m}^{-2}$ (average of 3 sites) } \\
\hline Anisantha rigida (Roth) Hyl. & 6 & 1 & 1 & 5 & 1 & 1 & 13 & 0 & 3 \\
\hline Melilotus sulcatus Desf. & 80 & 9 & 2 & 8 & 1 & 0 & 0 & 0 & 0 \\
\hline Hordeum vulgare L. (barley) & 0 & 0 & 0 & 52 & 56 & 37 & 15 & 1 & 1 \\
\hline Calendula stellata Cav. & 104 & 1 & 2 & 0 & 0 & 0 & 0 & 0 & 0 \\
\hline Scorpiurus muricatus L. & 2 & 10 & 14 & 0 & 1 & 0 & 1 & 1 & 2 \\
\hline Avena sterilis $\mathrm{L}$. & 0 & 7 & 18 & 0 & 0 & 0 & 0 & 0 & 0 \\
\hline Papaver rhoeas L. & 6 & 5 & 1 & 2 & 0 & 0 & 0 & 1 & 0 \\
\hline Arisarum vulgare Targ.-Tozz. & 11 & 5 & 0 & 1 & 0 & 1 & 2 & 4 & 0 \\
\hline Cichorium intybus $\mathrm{L}$. & 30 & 4 & 1 & 2 & 1 & 0 & 1 & 0 & 0 \\
\hline Lolium rigidum Gaud. & 56 & 8 & 1 & 0 & 1 & 0 & 0 & 0 & 0 \\
\hline Pisum sativum (pea) & 0 & 0 & 0 & 44 & 34 & 18 & 0 & 0 & 0 \\
\hline Andryala integrifolia $\mathrm{L}$. & 50 & 9 & 1 & 0 & 0 & 0 & 0 & 0 & 0 \\
\hline Lysimachia arvensis (L.) U.M. \& A. & 5 & 7 & 0 & 0 & 0 & 0 & 0 & 0 & 0 \\
\hline Rhagadiolus stellatus (L.) Gaertn. & 1 & 2 & 2 & 3 & 2 & 1 & 0 & 3 & 0 \\
\hline Glebionis coronaria $(\mathrm{L}$.$) Spach$ & 19 & 2 & 2 & 0 & 0 & 0 & 0 & 0 & 0 \\
\hline Geropogon hybridus L. & 2 & 4 & 3 & 0 & 1 & 1 & 0 & 1 & 1 \\
\hline Silene vulgaris (Moench) Garcke & 2 & 0 & 0 & 1 & 0 & 0 & 0 & 3 & 0 \\
\hline Vaccaria hispanica (Mill.) Rauschert & 1 & 2 & 1 & 1 & 0 & 0 & 1 & 0 & 0 \\
\hline Malva parviflora $\mathrm{L}$. & 26 & 2 & 0 & 0 & 0 & 0 & 0 & 0 & 0 \\
\hline Diplotaxis tenuisiliqua Delile & 2 & 2 & 2 & 0 & 0 & 0 & 3 & 0 & 0 \\
\hline Cynodon dactylon (L.) Pers. & 0 & 1 & 0 & 0 & 1 & 0 & 0 & 0 & 0 \\
\hline Sonchus oleraceus L. & 9 & 0 & 1 & 3 & 1 & 0 & 0 & 0 & 0 \\
\hline Convolvulus althaeoides $\mathrm{L}$. & 1 & 0 & 0 & 1 & 0 & 0 & 2 & 0 & 1 \\
\hline Sinapis arvensis $\mathrm{L}$. & 10 & 0 & 0 & 2 & 0 & 0 & 0 & 0 & 0 \\
\hline Ecballium elaterium (L.) A. Richard & 0 & 0 & 0 & 0 & 0 & 0 & 2 & 0 & 0 \\
\hline Bupleurum lancifolium Hornem. & 0 & 1 & 0 & 0 & 0 & 0 & 0 & 0 & 0 \\
\hline Vicia sativa $\mathrm{L}$. & 0 & 1 & 4 & 0 & 1 & 0 & 1 & 1 & 0 \\
\hline Medicago polymorpha $\mathrm{L}$. & 2 & 0 & 0 & 0 & 0 & 0 & 0 & 0 & 0 \\
\hline Triticum aestivum L. (bread wheat) & 3 & 0 & 0 & 0 & 0 & 0 & 0 & 0 & 0 \\
\hline Misopates orontium (L.) Raf. & 6 & 0 & 0 & 0 & 0 & 0 & 0 & 0 & 0 \\
\hline Convolvulus arvensis $\mathrm{L}$. & 2 & 0 & 0 & 0 & 0 & 0 & 0 & 0 & 0 \\
\hline Plantago afra $\mathrm{L}$. & 1 & 0 & 0 & 0 & 0 & 0 & 0 & 0 & 1 \\
\hline Silybum marianum (L.) Gaertn. & 1 & 0 & 0 & 0 & 0 & 0 & 1 & 0 & 0 \\
\hline Euphorbia exigua $\mathrm{L}$. & 6 & 0 & 0 & 0 & 0 & 0 & 0 & 0 & 0 \\
\hline Galium verrucosum Huds. & 3 & 1 & 0 & 0 & 0 & 0 & 0 & 0 & 0 \\
\hline Emex spinosa (L.) Campd. & 5 & 1 & 0 & 0 & 0 & 0 & 0 & 0 & 0 \\
\hline Phalaris minor Retz. & 0 & 1 & 0 & 0 & 0 & 0 & 0 & 0 & 1 \\
\hline Hippocrepis multisiliquosa $\mathrm{L}$. & 11 & 0 & 0 & 0 & 0 & 0 & 0 & 0 & 0 \\
\hline Teucrium resupinatum Desf. & 5 & 0 & 1 & 0 & 0 & 0 & 0 & 0 & 0 \\
\hline Other weeds & 21 & 1 & 2 & 3 & 0 & 0 & 0 & 0 & 0 \\
\hline Total $\left(\right.$ plants $\mathrm{m}^{-2}$ ) & 489 & 87 & 59 & 128 & 101 & 59 & 42 & 15 & 10 \\
\hline Standard error & 329 & 26 & 17 & 15 & 10 & 10 & 3 & 2 & 1 \\
\hline
\end{tabular}


Table 5. Weed density in no-till crops in rotation 3 (canola/durum wheat/durum wheat) in Chaouia from 2012-13 to $2014-15$

\begin{tabular}{|c|c|c|c|c|c|c|c|c|c|}
\hline \multirow{6}{*}{ Weed species } & \multicolumn{9}{|c|}{ Rotation 3: canola/durum wheat/durum wheat } \\
\hline & \multicolumn{3}{|c|}{$\begin{array}{c}\text { Canola } \\
\text { in 2012-13 }\end{array}$} & \multicolumn{3}{|c|}{$\begin{array}{c}\text { Durum wheat } \\
\text { in 2013-14 }\end{array}$} & \multicolumn{3}{|c|}{$\begin{array}{c}\text { Durum wheat } \\
\text { in } 2014-15\end{array}$} \\
\hline & & & & \multicolumn{6}{|c|}{ Date of weed density measurement } \\
\hline & $1 \mathrm{Nov}$ & 17 Jan & 2 Mar & $24 \mathrm{Dec}$ & $9 \mathrm{Feb}$ & 8 Mar & $24 \mathrm{Nov}$ & 15 Jan & $1 \mathrm{Apr}$ \\
\hline & 2012 & 2013 & 2013 & 2013 & 2014 & 2014 & 2014 & 2015 & 2015 \\
\hline & \multicolumn{9}{|c|}{ Plants $\mathrm{m}^{-2}$ (average of 3 sites) } \\
\hline Anisantha rigida (Roth) Hyl. & 0 & 0 & 0 & 1 & 1 & 2 & 0 & 0 & 4 \\
\hline Melilotus sulcatus Desf. & 3 & 2 & 1 & 2 & 0 & 0 & 0 & 11 & 0 \\
\hline Hordeum vulgare (barley) & 1 & 0 & 0 & 0 & 0 & 0 & 8 & 0 & 0 \\
\hline Calendula stellata Cav. & 2 & 1 & 0 & 0 & 0 & 0 & 0 & 1 & 0 \\
\hline Scorpiurus muricatus L. & 2 & 21 & 10 & 0 & 0 & 0 & 1 & 0 & 0 \\
\hline Papaver rhoeas $\mathrm{L}$. & 1 & 2 & 7 & 3 & 0 & 0 & 0 & 1 & 0 \\
\hline Arisarum vulgare Targ.-Tozz. & 5 & 4 & 4 & 0 & 3 & 2 & 1 & 1 & 0 \\
\hline Cichorium intybus $\mathrm{L}$. & 3 & 0 & 0 & 0 & 0 & 0 & 1 & 0 & 1 \\
\hline Lysimachia arvensis (L.) U.M.\&A. & 1 & 1 & 1 & 0 & 0 & 0 & 0 & 0 & 0 \\
\hline Rhagadiolus stellatus (L.) Gaertn. & 1 & 11 & 3 & 0 & 0 & 0 & 0 & 1 & 1 \\
\hline Geropogon hybridus $\mathrm{L}$. & 0 & 15 & 1 & 0 & 0 & 0 & 0 & 0 & 0 \\
\hline Silene vulgaris (Moench) Garcke & 3 & 0 & 0 & 0 & 11 & 0 & 3 & 0 & 0 \\
\hline Vaccaria hispanica (Mill.) Rauschert & 2 & 5 & 10 & 0 & 1 & 0 & 0 & 0 & 0 \\
\hline Malva parviflora $\mathrm{L}$. & 0 & 0 & 0 & 0 & 0 & 0 & 0 & 0 & 0 \\
\hline Diplotaxis tenuisiliqua Delile & 0 & 0 & 0 & 0 & 0 & 0 & 1 & 3 & 0 \\
\hline Cynodon dactylon (L.) Pers. & 1 & 3 & 1 & 3 & 4 & 4 & 3 & 1 & 0 \\
\hline Sonchus oleraceus L. & 3 & 0 & 1 & 1 & 0 & 0 & 0 & 0 & 0 \\
\hline Convolvulus althaeoides $\mathrm{L}$. & 2 & 2 & 1 & 1 & 0 & 0 & 0 & 0 & 0 \\
\hline Ecballium elaterium (L.) A. Richard & 2 & 1 & 1 & 1 & 1 & 1 & 3 & 1 & 0 \\
\hline Bupleurum lancifolium Hornem. & 0 & 0 & 0 & 0 & 3 & 0 & 0 & 0 & 0 \\
\hline Vicia sativa $\mathrm{L}$. & 1 & 2 & 0 & 0 & 1 & 0 & 0 & 1 & 0 \\
\hline Medicago polymorpha $\mathrm{L}$. & 0 & 0 & 0 & 0 & 0 & 0 & 0 & 1 & 0 \\
\hline Triticum aestivum L. (bread wheat) & 11 & 1 & 0 & 0 & 0 & 0 & 0 & 0 & 0 \\
\hline Misopates orontium (L.) Raf. & 2 & 0 & 2 & 0 & 0 & 0 & 0 & 0 & 0 \\
\hline Plantago afra $\mathrm{L}$. & 0 & 0 & 0 & 1 & 0 & 0 & 0 & 0 & 0 \\
\hline Silybum marianum (L.) Gaertn. & 1 & 0 & 0 & 0 & 0 & 0 & 2 & 0 & 0 \\
\hline Euphorbia exigua L. & 0 & 1 & 2 & 0 & 0 & 0 & 0 & 0 & 0 \\
\hline Emex spinosa (L.) Campd. & 1 & 0 & 0 & 0 & 0 & 0 & 0 & 0 & 0 \\
\hline Phalaris minor Retz. & 1 & 3 & 1 & 0 & 0 & 0 & 0 & 0 & 0 \\
\hline Teucrium resupinatum Desf. & 1 & 0 & 0 & 0 & 0 & 0 & 0 & 0 & 0 \\
\hline Others & 8 & 7 & 2 & 7 & 2 & 1 & 2 & 0 & 0 \\
\hline Total $\left(\right.$ plants $\mathrm{m}^{-2}$ ) & 58 & 82 & 49 & 20 & 27 & 12 & 25 & 22 & 6 \\
\hline Standard error & 9 & 32 & 9 & 3 & 5 & 4 & 2 & 7 & 5 \\
\hline
\end{tabular}

\subsection{Weed Diversity in Barley + Pea Fields}

In forage mixture barley + pea, weed densities were 489, 87, and 59 plants $\mathrm{m}^{-2}$ in November 2012, January 2013 , and April 2013 few days before haying, respectively (Table 4). By using pre-plant glyphosate, planting no-till herbicide-free forage mixture barley + pea would decrease selection pressure for herbicide resistance. Furthermore, barley + pea mixture was an appropriate choice as a crop in crop/livestock integrated dryland production systems because of its potential for high yield and competitiveness with weeds. Barley and pea are well adapted to semiarid regions, and may be a good approach for forage production and weed management. Indeed, barley + pea produce good yields with nutritive values suitable for sheep and cattle (Carr et al., 2004; Lenssen, 2008). 
Table 4 shows that major weed species associated to barley + pea mixture were Anisantha rigida, Avena sterilis, Calendula stellata, Cichorium intybus, Glebionis coronaria, Lolium rigidum, Lysimachia arvensis, Melilotus sulcata, Papaver rhoeas, and Scorpiurus muricatus. All these weeds were palatable and not toxic to livestock.

\subsection{Weed Diversity in Canola Fields}

In 2012-13, weed densities were 58 plants $\mathrm{m}^{-2}$ before glyphosate application at planting (Table 5 ). Weed density

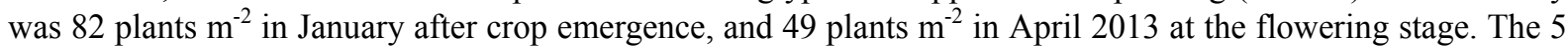
major weed species associated to canola were Geropogon hybridus, Rhagadiolus stellatus, Scorpiurus muricatus, Vaccaria hispanica, and volunteer wheat. Such weeds are common in rainfed canola fields in Morocco (Tanji \& El Brahli, 2017).

\section{Conclusion}

This study showed that the initial soil weed seedbank was 1890 seeds $\mathrm{m}^{-2}$. Seedbank reductions after two cropping seasons were $23 \%$ in continuous durum wheat, $68 \%$ in the durum wheat after canola, and $72 \%$ in durum wheat after barley + pea mixture. Such reductions were due to glyphosate use before no-till planting, post-emergence herbicide application in durum wheat, mowing barley + pea mixture for hay. Continuous durum wheat increased rigid brome (Anisantha rigida) density and seedbank. For a good integration crop/livestock production system, grazed weedy fallow should be replaced by barley + pea forage mixture. This study demonstrated the combined merits of pre-plant glyphosate, herbicide use in durum wheat, herbicide-free barley + pea haying, and durum wheat rotation with either canola or barley + pea to manage weeds in no-till systems in semi-arid Morocco. Further research is needed concerning the effects of the weed-suppressive potential of longer (> 3 years) rotations.

\section{Acknowledgements}

The authors thank farmer collaborators for their help and contributions. The financial assistance of the ACLIMAS (Adaptation to Climate Change of the Mediterranean Agricultural Systems) project within the Sustainable Water Integrated Management (SWIM) programme of the European Commission is gratefully acknowledged. Fee publication has been paid by Mohamed El Mourid, ICARDA regional coordinator (North Africa Program).

\section{References}

Abail, Z., El Gharous, M., Belmekki, M., \& Iben Halima, O. (2013). Conservation agriculture and its impact on soil quality: Highlights of Moroccan research results in semi-arid areas. Engineering Science and Technology, 3(4), 699-703.

Aibar, J. (2006). La lutte contre les mauvaises herbes pour les céréales en semis direct: Principaux problèmes. Options Méditerranéennes, Série A, 69, 19-26.

Anderson, R. L. (2015). Integrating a complex rotation with no-till improves weed management in organic farming: A review. Agronomy for Sustainable Development, 35(3), 967-974. https://doi.org/10.1007/ s13593-015-0292-3

Belmekki, M., Mrabet, R., El Gharous, M., Moussadek, R., Iben Halima, O., Boughlala, M., \& Bencharki, B. (2014). Effets du non labour et de la gestion des résidus sur la conservation de l'eau et la qualité d'un sol calcimagnésique dans les zones semi-arides Marocaines. International Journal of Innovation and Applied Studies, 6(4), 792-800. Retrieved from http://www.ijias.issr-journals.org

Blackshaw, R. E., Moyer, J. R., Harker, K. N., \& Clayton, G. W. (2005). Integration of agronomic practices and herbicides for sustainable weed management in a zero-till barley field pea rotation. Weed Technology, 19(1), 190-196. https://doi.org/10.1614/WT-04-128R

Blackshaw, R. E., Pearson, D. C., Larney, F. J., Regitnig, P. J., Nitschelm, J. J., \& Lupwayi, N. Z. (2015). Conservation management and crop rotation effects on weed populations in a 12-year irrigated study. Weed Technology, 29(4), 835-843. https://doi.org/10.1614/WT-D-15-00071.1

Boughlala, M., El Gharras, O., \& Dahan, R. (2011). Economic comparison between conventional and no-tillage farming systems in Morocco. Hommes, Terre et Eaux, 149-150, 35-39.

Carr, P. M., Horsley, R. D., \& Poland, W. W. (2004). Barley, oat, and cereal-pea mixtures as dryland forages in the northern Great Plains. Agronomy Journal, 96(3), 677-684. https://doi.org/10.2134/agronj2004.0677 
El Brahli, A., \& Mrabet, R. (2000). La jachère chimique pour relancer la céréaliculture non irriguée en milieu semi-aride marocain. Proceedings de la journée nationale sur le désherbage des céréales, Association Marocaine de Malherbologie, Settat (pp. 133-145).

El Gharras, O., El Hantaoui, N., \& El Brahli, A. (2010). Le semis direct dans la Chaouia. Agriculture du Maghreb, 46, 90-93.

El Housni, A., El Maadoudi, A. H., \& Bendaou, M. (2013). Stratégies d'amélioration de la valeur nutritionnelle des jachères au Maroc. Options Méditerranéennes, A 108, 115-120.

Friedrich, T. (2005). Herbicides and no-till farming: Does no-till farming require more herbicides? Outlooks on Pest Management, August 2005, 188-191.

Gulden, R. H., Lewis, D. W., Froese, J. C., Van Acker, R. C., Martens, G. B., Entz, M. H., ... Bell, L. W. (2011). The effect of rotation and in-crop weed management on the germinable weed seedbank after 10 years. Weed Science, 59(4), 553-561. https://doi.org/10.1614/WS-D-11-00001.1

Hamal, A., Rzozi, S. B., Benbella, M., Msatef, Y., \& Bouhache, M. (2001). Evaluation of the seedbank and dynamics of rigid brome (Bromus rigidus Roth) in wheat in the Sais area, Morocco. Meded Rijksuniv Gent Fak Landbouwkd Toegep Biol Wet., 66(2b), 753-759.

Harker, K. N., \& O'Donovan, J. T. (2013). Recent weed control, weed management, and integrated weed management. Weed Technology, 27(1), 1-11. https://doi.org/10.1614/WT-D-12-00109.1

Holm, F. A., Zentner, R. P., Thomas, A. G., Sapsford, K., Légère, A., Gossen, B. D., ... Leeson, J. Y. (2006). Agronomic and economic responses to integrated weed management systems and fungicide in a wheat-canola-barley-pea rotation. Canadian Journal of Plant Science, 86(4), 1281-1295. https://doi.org/ 10.4141/P05-165

Jose Maria, L., \& Sans, F. X. (2011). Weed seedbanks in arable fields: Effects of management practices and surrounding landscape. Weed Research, 51(6), 631-640. https://doi.org/10.1111/j.1365-3180.2011.00872.x

Kassam, A. (2014). Overview of the current status of conservation agriculture globally and challenges with designing and adapting CA to the circumstances of the smallholders (pp. 2-4). Regional Conference on Conservation Agriculture for Smallholders in Asia and Africa, December 7-11, 2014, Mymensigh, Bangladesh.

Labbaci, T., Dugué, P., Kemoun, H., \& Rollin, D. (2015). Innovation et action collective: Le semis direct des cultures pluviales au Moyen Sebou (Maroc). Cahiers Agricultures, 24(2), 76-83. https://doi.org/10.1684/ agr.2015.0742

Lenssen, A. W. (2008). Planting date and preplant weed management influence yield, water use, and weed seed production in herbicide-free forage barley. Weed Technology, 22(3), 486-492. https://doi.org/10.1614/ WT-08-009.1

Loss, S., Haddad, A., Desbioles, J., Cicek, H., Khalil, Y., \& Piggin, C. (2015). The practical implementation of conservation agriculture in the Middle East (100 pages). ICARDA (International Center for Agricultural Research in the Dry Areas), ACIAR (Australian Center for International Agricultural Research).

Lutman, P. J. W., Cussans, G. W., Wright, K. J., Wilson, B. J., Wright, G. M., \& Lawson, H. M. (2002). The persistence of seeds of 16 weed species over six years in two arable fields. Weed Research, 42(3), 231-241. https://doi.org/10.1046/j.1365-3180.2002.00281.x

Moussadek, R. (2011). Impacts de l'agriculture de conservation sur les propriétés et la productivité des vertisols du Maroc Central. Africa Focus, 25(2), 147-151.

Mrabet, R. (2008). No tillage systems for sustainable dryland agriculture in Morocco (153 pages). INRA, Rabat.

Mrabet, R., Moussadek, R., Fadlaoui, A., \& Van Ranst, E. (2012). Conservation agriculture in dry areas of Morocco. Field Crops Research, 132, 84-94.

Murphy, S. D., Clements, D. R., Belaoussoff, S., Kevan, P. G., \& Swanton, C. J. (2006). Promotion of weed species diversity and reduction of weed seedbanks with conservation tillage and crop rotation. Weed Science, 54(1), 69-77. https://doi.org/10.1614/WS-04-125R1.1

Nichols, V., Verhulst, N., Cox, R., \& Govaerts, B. (2015). Weed dynamics and conservation agriculture principles: A review. Field Crops Research, 183, 56-68. https://doi.org/10.1016/j.fcr.2015.07.012 
Ramdani, A., Mrabet, R., \& Gomez Macpherson, H. (2010). Performance of Moroccan wheat cultivars under tillage systems and water regimes. Journal of Agricultural Science and Technology, 4(5), 106-111.

Recasens, J., García, A. L., Cantero-Martínez, C., Torra, J., \& Royo-Esnal, A. (2016). Long-term effect of different tillage systems on the emergence and demography of Bromus diandrus in rainfed cereal fields. Weed Research, 56(1), 31-40. https://doi.org/10.1111/wre.12185

Schwilch, G., Laouina, A., Chaker, M., Machouri, N., Sfa, M., \& Stroosnijder, L. (2015). Challenging conservation agriculture on marginal slopes in Sehoul, Morocco. Renewable Agriculture and Food Systems, 30, 233-251. https://doi.org/10.1017/S1742170513000446

Smith, R. G., \& Gross, K. L. (2006). Rapid change in the germinable fraction of the weed seedbank in crop rotations. Weed Science, 54(6), 1094-1100. https://doi.org/10.1614/WS-06-090R.1

Stone, J. C., Peeper, T. F., \& Stone, A. E. (2006). Rotational cropping systems to reduce cheat (Bromus secalinus) densities. Weed Technology, 20, 445-452. https://doi.org/10.1614/WT-05-107R1.1

Tanji, A., \& El Brahli, A. (2017). Weed surveys in rainfed canola (Brassica napus L.) fields in Chaouia, Morocco. European Journal of Agriculture and Forestry Research, 5(3), 1-15.

Tanji, A., El Gharras, O., Mayfield, A., \& El Mourid, M. (2017). On-farm evaluation of integrated weed management in no-till rainfed crops in semi-arid Morocco. African Journal of Agricultural Research, 12(16), 1404-1410. https://doi.org/10.5897/AJAR2017.12248

Tuesca, D., Puricelli, E., \& Papa, J. (2001). A long-term study of weed flora shifts in different tillage systems. Weed Research, 4l(4), 369-382. https://doi.org/10.1046/j.1365-3180.2001.00245.x

\section{Copyrights}

Copyright for this article is retained by the author(s), with first publication rights granted to the journal.

This is an open-access article distributed under the terms and conditions of the Creative Commons Attribution license (http://creativecommons.org/licenses/by/4.0/). 